\title{
Non-invasive diagnosis of portal hypertension in cirrhosis using ultrasound based elastography
}

\author{
Ivica Grgurevic ${ }^{1}$, Tomislav Bokun ${ }^{1}$, Tonci Bozin ${ }^{1}$, Vladimir Matic ${ }^{1}$, Sara Haberle ${ }^{1}$, Ioan Sporea ${ }^{2}$
}

${ }^{1}$ Department of Gastroenterology, Hepatology and Clinical Nutrition, University Hospital Dubrava, University of Zagreb School of Medicine and Faculty of Pharmacy and Biochemistry, Zagreb, Croatia, ${ }^{2}$ Department of Gastroenterology and Hepatology, "Victor Babeș“ University of Medicine and Pharmacy Timişoara, Romania.

\begin{abstract}
Liver stiffness measurement (LSM) by ultrasound-based elastography may be used to non-invasively discriminate between the stages of liver fibrosis, rule out cirrhosis and follow its evolution, including the prediction of the presence of oesophageal varices. The same is possible in order to diagnose clinically significant portal hypertension, referring primarily to transient elastography and LSM values $\geq 20-25 \mathrm{kPa}$. The same approach may be used to reliably rule out the presence of oesophageal varices (LSM $<20 \mathrm{kPa}+$ platelets $>150 \times 10^{9} / \mathrm{L}$ ). These recommendations refer primarily to patients with viral aetiology of chronic liver disease (hepatitis C), while additional studies are required for other aetiologies. While spleen stiffness measurement (SSM) also poses a logical choice in this indication, controversial results have nevertheless been published on this issue. It should be emphasized, however, that more recent data indicate that this parameter should be included in the diagnostic algorithm for portal hypertension, if not as the sole then as a part of a sequential algorithm, combined with LSM. Until now, transient elastography has been most extensively studied and founded on scientific evidence, although the results of other ultrasound-based elastography techniques demonstrate the same trend for the non-invasive assessment of portal hypertension.

Keywords: portal hypertension; ultrasound based elastography; liver stiffness; spleen stiffness
\end{abstract}

\section{Introduction}

Portal hypertension is defined as an increase in portal venous pressure leading to the development of portosystemic shunts and other complications, which result from the congestion of the organs drained by the portal venous system. The most prominent complications of portal hypertension are variceal bleeding, ascites, and portal encephalopathy. Depending on the anatomic location of the pathomorphological substrate responsible for its development, portal hypertension can be divided into post-hepatic, intra-hepatic and pre-hepatic causes. Intra-hepatic

Received Accepted

Med Ultrason

2017, Vol. 19, No 3, 310-317

Corresponding author: Ivica Grgurevic, MD, PhD, Assist. Prof. Department of Gastroenterology, Hepatology and Clinical Nutrition

University Hospital Dubrava

Avenija Gojka Suska 6, Zagreb 10 000, Croatia

Phone: +385 1290 2554, Fax: +38512902550

E-mail: ivica.grgurevic@zg.htnet.hr causes can additionally be divided into post-sinusoidal, sinusoidal and pre-sinusoidal causes [1].

Liver cirrhosis is the most common cause of portal hypertension, which results from increased portal vascular resistance in hepatic parenchyma due to the progressive accumulation of fibrous tissue and distortion of the normal liver architecture by regenerative nodules. Increased blood flow in the portal vein, as a result of splanchnic vasodilatation, contributes to portal pressure as well $[1,2]$. Splanchnic vasodilatation is a consequence of the abundant production of vasoactive, particularly vasodilating, substances (such as Nitric Oxide) in mesenterial lymph nodes, as a result of the ongoing low-intensity chronic inflammation initiated and promoted by intestinal bacterial translocation. Increased intestinal permeability is a result of venous blood congestion, together with oedema and the weakening of intercellular junctions as a consequence of compromised mucosal barrier. All factors combine to cause hyperdynamic circulation, which is a typical haemodynamic consequence of portal hypertension $[1,2]$. Analysing the natural course of patients with cirrhosis, 
D'Amico and colleagues confirmed the crucial influence of portal hypertension on the prognosis. Based on portal hypertensive complications, cirrhosis may be divided into four clinical stages: stage 1) no varices and ascites; stage 2 ) varices without ascites; stage 3 ) ascites with or without varices; and stage 4) variceal bleeding with or without ascites. The corresponding one-year mortality rates for these stages were $1 \%$ for stage $1,3.4 \%$ for stage $2,20 \%$ for stage 3 and a remarkable $57 \%$ for stage 4 [3].

The most exact method to measure portal pressure is the hepatic venous pressure gradient (HVPG) procedure $[4,5]$. To measure the HVPG, it is necessary to insert a transjugular catheter into the right hepatic vein to quantify wedged and free pressures. Wedged pressure is sinusoidal pressure, while free pressure results from both sinusoidal and retrograde pressure from the right atrium. The HVPG represents the difference between wedged and free pressure in hepatic veins. The normal HVPG value is up to $5 \mathrm{mmHg}$ and above $6 \mathrm{mmHg}$ is considered to be portal hypertension. Furthermore, portal hypertension can be divided into subclinical (HVPG 6-10 mmHg), as well as clinically significant portal hypertension (CSPH) with an HVPG value $\geq 10 \mathrm{mmHg}$ [5]. Complications, such as oesophageal varices, ascites, and bleeding, tend to occur at values $>10 \mathrm{mmHg}$. A value $>16 \mathrm{mmHg}$ implies a severe risk of death due tov ariceal bleeding [1-3].

The HVPG represents the most reliable prognostic indicator in patients with cirrhosis. It is more reliable than the Child-Pugh and MELD classifications, which underscore the significance of portal hypertension in the natural progression of cirrhosis and its complications [6]. With this in mind, the HVPG is tremendously important for prognostication and guiding therapeutic decisions. It has been shown that patients with compensated cirrhosis and CSPH had higher mortality rates after liver resection due to hepatocellular carcinoma in comparison with patients with an HVPG value $<10 \mathrm{mmHg}[7,8]$.

Considering that the technique of measuring the HVPG is invasive and, therefore, accompanied by certain periprocedural risks, as well as only being available in a small number of centres, there is an ongoing search for non-invasive methods, which could be reliably used to detect and stage portal hypertension and its complications and replace the HVPG procedure. Among the noninvasive imaging methods ultrasound is usually the first to be used in patients with chronic liver disease. Different ultrasound-derived parameters including the portal vein diameter, presence of porto-systemic collaterals, spleen size, congestion index of the portal vein, portal hipertensive index as well as liver stiffness have already proved valuable for diagnosis of esophageal varices as the typical complication of portal hypertension [9]. In the following text, ultrasound based elastography will be reviewed as a non-invasive method for the evaluation of portal hypertension in patients with liver cirrhosis.

\section{Ultrasound based elastography}

Ultrasound based elastography is a method that provides diagnostic information on tissue stiffness, in this case, liver and/or spleen and can be divided into qualitative and quantitative approaches, and into strain elastography and shear wave elastography (SWE), depending on the technology available [10-11]. Strain elastography measures relative tissue stiffness as compred to the reference tissue spot, in real time.

The main principle of quantitative ultrasound elastography is based on measuring the velocity of shear waves induced in the investigated tissue by the tissue deformation, which results from mechanical or acoustic force [10]. The magnitude of the deformation is inversely related to the tissue stiffness, whereas the velocity of the shear waves is proportional.

By introducing shear wave speeds into Young's modulus of elasticity equation, the obtained value in $\mathrm{kPa}$ represents the tissue stiffness. There are several approaches to SWE. The first is Transient Elastography (TE), which is a mono-dimensional method that uses a mechanical vibrator with a coaxially coupled pulse Doppler probe. Furthermore, as this method does not have the ability to provide 2D images of the liver, it only shows the elastogram and the quantitative value of the liver stiffness measurements (LSM) (fig 1a). However, this method is very simple to perform and widely accepted in the hepatological community, given the large body of scientific evidence confirming its effectiveness. Another version is based on the transmission of an increased acoustic impulse known as an acoustic radiation force impulse imaging (ARFI), which is generated by the modified conventional convex abdominal ultrasound probe. This method enables $2 \mathrm{D}$ imaging of the liver, coupled by the stiffness measurement in the region of interest within the liver as chosen by operator, and some of them display elastograms in real time as well. Depending on the presence of these features, methods based on the ARFI can be furtherly divided into point shear wave elastography (pSWE) and 2D shear wave elastography (2DSWE) $[10,11]$ (fig $1 \mathrm{~b}, \mathrm{c})$. Many manufacturers have introduced ARFI technology to their ultrasound machines in the form of pSWE, such as Siemens' Virtual Touch tissue quantification (VTTQ) and Philips' elasticity point quantification (ElastPQ). Among several manufacturers that use 2D-SWE, namely, Supersonic Imagine (SSI), General Electric, Toshiba, only SSI has so far been sufficiently evaluated for the non-invasive diagnosis of liver disease. 


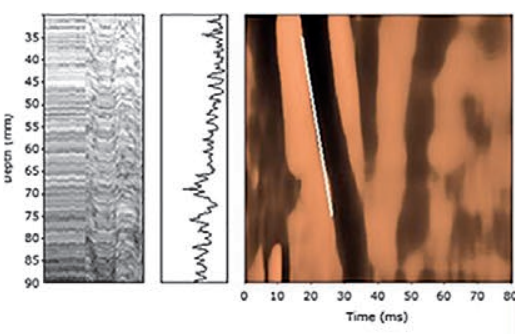

CAP

a) $330 \mathrm{~dB} / \mathrm{m}$
E

$55.1 \mathrm{kPa}$

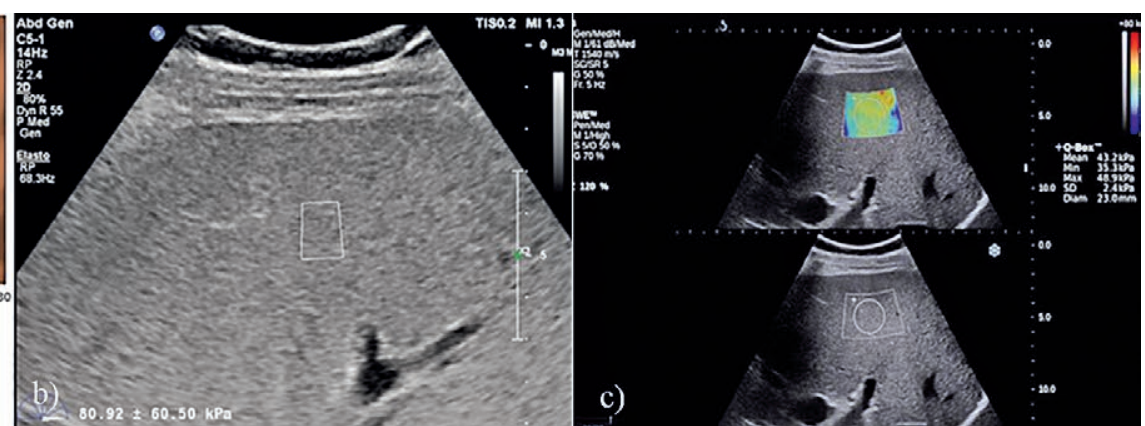

Fig 1. Representative images of liver stiffness measurements (LSM) in patients with clinically significant portal hypertension as obtained by Transient Elastography (A), Point Shear Wave Elastography (B) and Two Dimensional Shear Wave Elastography (C).

In chronic liver disease, the main histological feature is the accumulation of connective tissue. This results in a harmful process, which occurs in the liver and leads to necrosis/apoptosis of hepatic or biliary cells [12]. Accumulation of connective tissue makes the liver stiffer, which can be quantified by ultrasound based elastography. During the last 10 years, elastography is widely accepted as a non-invasive method to assess the stage of liver fibrosis following numerous studies conducted among patients with chronic viral hepatitis $\mathrm{C}$ and $\mathrm{B}$ [13]. Elastography is particularly reliable when it comes to ruling-in significant fibrosis (stage $\mathrm{F} \geq 2$ to METAVIR classification) and the ruling-out of cirrhosis (stage $\mathrm{F}=4$ to METAVIR). However, once cirrhosis presents, the pathophysiological processes in the liver are underway in the molecular, histological, macroscopic and clinical phenotypes $[3,12,14,15]$. It is well known that cirrhosis can be divided into clinical stages, according to the Child-Pugh classification. From a pathological perspective, histological changes accompany the worsening of portal hypertension, while oesophageal varices develop, causing liver failure, followed by the accumulation of ascites, variceal bleeding, portal encephalopathy, jaundice, and failure of other liver functions [2,3,13]. All of these clinical manifestations have a histological basis, whereas one of the most significant findings is progressive accumulation and chemical structure changes of the connective tissue in the liver, which becomes more rigid with the formation of acellular scars. Importantly, progressive liver cirrhosis results in the liver becoming stiffer, which can be measured elastographically (this is the basis for the clinical application of ultrasound elastography) [15].

\section{Ultrasound based elastography in portal hypertension. General principles}

Important research regarding the relationship between the portal hypertension value and elastographi- cally estimated liver stiffness was reported by a group of authors from Florence in 2007 [16]. The study included 61 patients with compensated cirrhosis as a result of chronic hepatitis $\mathrm{C}$ aetiology. The findings showed a good correlation $(r=0.81, p<0.0001)$ between the LSM evaluated with TE and the HVPG. However, further analysis showed that patients can be divided into two groups: one with the values of $\mathrm{HVPG}<10-12 \mathrm{mmHg}$, in which the correlation between the HVPG and the LSM in linear regression analysis was very good $\left(\mathrm{r}^{2}=0.72, \mathrm{p}<0.0001\right)$; and the other, with values of $>10-12 \mathrm{mmHg}$ with poor correlation $\left(\mathrm{r}^{2}=0.35, \mathrm{p}<0.0001\right.$ and $\left.\mathrm{r}^{2}=0.17, \mathrm{p}=0.02\right)$. This phenomenon is explained by the fact that portal hypertension in the early phase results from the increased resistance to blood flow within the fibrotic liver, and these fibrotic changes translate to the increased liver stiffness. With further aggravation of portal hypertension it becomes more dependent on extra-hepatic factors, such as increased splanchnic blood flow (antegrade component of $\mathrm{PH}$ ), as well as the dynamic component within the liver due to endothelial dysfunction in cirrhosis and hepatic stellate cell contraction around the sinusoids. These last two factors cannot be measured by elastography, which is why worsening portal hypertension results in the loss of any correlation between the LSM and the HVPG. Since the values of HVPG $>10 \mathrm{mmHg}$ are those that define $\mathrm{CSPH}$, it follows that the elastographic measurement of liver stiffness can be used to differentiate patients with or without CSPH, but cannot determine the severity of portal hypertension above these values, including the monitoring of the response to treatment with non-selective beta blockers.

\section{Transient Elastography in portal hypertension}

Since Transient Elastography (TE) first appeared on the market, it has experienced widespread use in clinical hepatology, primarily due to its ease of handling and re- 
liability, as well as the reproducibility of results [17-19]. A meta-analysis of 26 studies involving 3,644 patients dealt with the research reliability of TE in distinguishing patients with or without CSPH and those with or without oesophageal varices, as the key manifestation of portal hypertension, including the detection of high-risk oesophageal varices [20]. In distinguishing patients with and without CSPH, TE had sensitivity of $90 \%$, a specificity of $79 \%$, a positive predictive value (PPV) of $88 \%$ and a negative predictive value (NPV) of $88 \%$. However, since the value of the PPV and NPV significantly depends on the prevalence of the investigated condition, while the approximate prevalence of CSPH in the studies in this meta-analysis was $50-75 \%$, it was shown that the probability of the correct classification of patients in the CSPH group was $81-93 \%$ in the case of LSM values above the defined threshold for CSPH. At the same time, in $11-27 \%$ of cases with LSM values below the threshold, CSPH patients will be incorrectly classified. In other words, in spite of the negative measurement, patients will have CSPH. Therefore, LSM by TE can be used to confirm $\mathrm{CSPH}$, but not to exclude its existence. Reverse results were obtained in patients with oesophageal varices. With regard to the detection of oesophageal varices (any grade vs. no varices), the LSM had a sensitivity of $87 \%$, a specificity of $53 \%$, a PPV of $79 \%$ and an NPV of $64 \%$. With an average prevalence of oesophageal varices at about $50 \%$ in the included studies, the probability for the correct classification of patients following a positive measurement (above the threshold) was only $65 \%$, while $20 \%$ of oesophageal varices were missed following a negative measurement. In large oesophageal varices with a prevalence of $25 \%$ in the included studies, TE correctly identified $41 \%$ of patients following a positive LSM (above the threshold), whereas only $7 \%$ of cases were missed following the negative measurements below the threshold. In other words, TE can reliably rule-out large oesophageal varices in cases of LSM which are below the defined threshold, but it is not reliable for identifying oesophageal varices (any stage including large varices).

Llop et al [21] analysed 97 patients with resected primary hepatocellular carcinoma and cirrhosis in the Child-PughA stage. All patients underwent TE of the liver and HVPG measurement. Using a previously defined LSM cut-off values reliable to rule-out $(<13.6 \mathrm{kPa})$ and rule-in $(21.1 \mathrm{kPa}) \mathrm{CSPH}$, the authors correctly classified $53 \%$ of patients, while $47 \%$ remained in the 'grey zone' between these values.

Augustin et al [22] analysed 250 patients with chronic liver disease who underwent liver TE, as well as ultrasound of the liver, spleen and a determination of the platelets' count. Patients with an LSM $>13.6 \mathrm{kPa}$ were offered an endoscopic examination in order to determine the existence of oesophageal varices. Patients were divided into the groups: a) patients with a normal ultrasound appearance of the liver, spleen and normal platelet count $\left(>150 \times 10^{9} / \mathrm{L}\right)$; b) patients with a normal ultrasound appearance of the liver and spleen, but decreased platelet count $\left(<150 \times 10^{9} / \mathrm{L}\right.$; and $\left.\mathrm{c}\right)$ patients with pathological ultrasound findings of the liver (nodularity) or spleen (splenomegaly) and a decreased platelet count. An LSM $>13.6$ $\mathrm{kPa}$ was found in $8 \%, 43 \%$ and $81 \%$ of patients in groups a,b and c, respectively, whilst oesophageal varices were found, again respectively, in $0 \%, 6 \%$ and $43 \%$ of patients in these groups. The LSM optimal critical value for determining CSPH was $25 \mathrm{kPa}$ (PPV 94\%, NPV 59\%), while the optimal value for ruling-out the presence of oesophageal varices was $21.1 \mathrm{kPa}$ (PPV 33\%, NPV 92\%). Given these results, it can be concluded that LSM values above $25 \mathrm{kPa}$ reliably indicate the presence of CSPH. However, in the case of LSM values in the range of $13.6-25 \mathrm{kPa}$, along with a normal liver and spleen ultrasound, as well as a normal platelet count $>150 \times 10^{9} / \mathrm{L}$ the risk of large oesophageal varices is $0 \%$.

Ding NS et al investigated the diagnostic reliability of the combination of an LSM of $25 \mathrm{kPa}$ and a platelet count of $<100 \times 10^{9} / \mathrm{L}$ in the detection of high-risk gastroesophageal varices in 271 patients with Child-Pugh A cirrhosis [23]. In patients with an $\mathrm{LSM}<25 \mathrm{kPa}$ and a platelet count $>100$, none had high-risk varices, while they were found in $21 \%$ of patients with an LSM $>25 \mathrm{kPa}$ and a platelet count $<100$.

Meanwhile, Abraldes et al analysed 182 patients with compensated cirrhosis, of whom 109 (60\%) had CSPH, by measuring their HVPG ("Anticipate" study). They analysed the LSM values and the platelet counts [24]. Among patients with an LSM $>25 \mathrm{kPa}, 96 \%$ had CSPH. Among patients with an LSM $<25 \mathrm{kPa}$ and platelets $>150$, CSPH was present in $17 \%$, while it was present in $54 \%$ of patients with an LSM $<25 \mathrm{kPa}$ and platelets $<150$.

Taking into consideration the results of these studies, the Baveno VI Consensus Conference on portal hypertension issued a recommendation that TE may be used as a non-invasive method for determining CSPH if the LSM $>20-25 \mathrm{kPa}$. In addition, one may exclude the presence of large (high-risk) oesophageal varices in cases where LSM values are $<20 \mathrm{kPa}$, provided that the platelet count is $>150 \times 10^{9} / \mathrm{L}$. This recommendation primarily refers to the viral (hepatitis $C$ ) aetiology of liver disease[25].

Since the release of the Baveno VI recommendations, they have been validated in independent studies involving patients with chronic liver disease and 
at risk of oesophageal varices. One of the largest studies included 395 patients with compensated advanced chronic liver disease (advanced fibrosis and compensated cirrhosis) [26]. LSM was performed using the $\mathrm{TE}$, with platelet values obtained and oesophageal endoscopy undertaken in order to detect oesophageal varices. Oesophageal varices were found in $119(29 \%)$ patients, with large oesophageal varices recorded in 35 (9\%) patients. By implementing the Baveno VI criteria, the authors managed to correctly identify $32 / 35$ (92\%) patients with large oesophageal varices using a noninvasive algorithm. Although three (8\%) patients were missed, it was nevertheless possible to avoid endoscopy in $28.1 \%$ of patients. In the meta-analysis of 15 studies (3,364 patients), which evaluated the Baveno VI recommendations, large oesophageal varices (with the risk of bleeding) were reliably excluded in $96 \%$ patients with an $\mathrm{LSM}$ value $<20 \mathrm{kPa}$ and platelets $>150 \times 10^{9} / \mathrm{L}$, with the remaining $4 \%$ of those who were wrongly classified [27].

\section{Spleen elastography in portal hypertension}

Since the venous blood from the splenic veins drains in the portal blood flow, it is logical to expect a change in the morphology and functions of the spleen in portal hypertension. Indeed, one of the most common signs of portal hypertension is splenomegaly, which is caused mainly by congestion of the spleen. It is noteworthy that, besides passive spleen congestion, significant histological changes, such as the accumulation of fibrous tissue, and the proliferation and hypertrophy of certain tissue elements in the spleen, have been identified in the studies dealing with portal hypertension [28]. This results in an increase in the spleen stiffness, which can be analysed by elastography. The meta-analysis of 12 studies with 1,497 patients found suboptimal diagnostic accuracy of spleen stiffness measurement (SSM) in the detection of oesophageal varices, with a sensitivity and specificity of $<80 \%$ [29]. Nevertheless, by using both LSM and SSM Stefanescu et al were able to predict the presence of oesophageal varices with accuracy of $89.95 \%$ [30]. Recent meta-analysis, which included 16 studies involving 1,892 patients undergoing LSM and SSM, reported a better overall diagnostic accuracy of SSM, compared with LSM, in the diagnosis of the presence (any grade) of oesophageal varices (AUROC 0.88 vs. $0.81, \mathrm{p}<0.01$ ). In this meta-analysis no significant difference in sensitivity between LSM and SSM existed ( 0.83 and 0.88 respectively, $\mathrm{p}=0.23$ ), whereas SSM was significantly more specific ( 0.78 vs. 0.66 for LSM, $p=0.02)$ for this indication[31].

\section{PointSWE (pSWE) in portal hypertension}

Of all the pSWE methods, in this indication, only VTTQ based on ARFI technology has been evaluated so far. In a study of 88 patients with cirrhosis, LSM was carried out using ARFI technology, with diagnostic reliability comparable to TE (cut-off value $2.58 \mathrm{~m} / \mathrm{s}$, AUROC 0.855 for ARFI and $16.8 \mathrm{kPa}$, AUROC0.870 for TE). In the detection of oesophageal varices, VTTQ had a worse performance with an AUROC of 0.743 (cut-off value $2.74 \mathrm{~m} / \mathrm{s}$ ) compared to TE (AUROC 0.802, cut-off value $9.27 \mathrm{kPa}$ ) [32].

In a South Korean study, based on 143 patients with compensated cirrhosis, using VTTQ, LSM cut-off values of 2.08 and $1.9 \mathrm{~m} / \mathrm{s}$ had a sensitivity of 64.9 and $85 \%$, and a specificity of 81.1 and $67.5 \%$ for the detection of any grade oesophageal varices and high-risk oesophageal varices [33].

Attia et al [34] evaluated the diagnostic accuracy of LSM and SSM when using ARFI technology (VTTQ) in 78 patients with chronic liver disease, of which $76 \%$ had oesophageal varices; all patients underwent HVPG measurement as well. With cut-off values of 2.17 and $2.32 \mathrm{~m} / \mathrm{s}$, LSM and SSM had a very good performance to diagnose CSPH (AUROCs 0.929 and 0.968, respectively). For the detection of patients with CSPH and oesophageal varices, diagnostic accuracy of LSM (cut-off value $2.29 \mathrm{~m} / \mathrm{s}$ ) and SSM (cut-off value $2.55 \mathrm{~m} / \mathrm{s}$ ) was 0.84 and 0.899 , respectively.

Conflicting results were obtained in terms of the diagnostic accuracy of spleen stiffness measurement (SSM) using ARFI technology (VTTQ) for the diagnosis of CSPH and/or oesophageal varices. In the study by Takuma et al [35], SSM was performed in 340 patients with liver cirrhosis, resulting in exceptionally high NPV $(98.4 \%$ at cut-off value $3.18 \mathrm{~m} / \mathrm{s})$ for the presence of any grade of oesophageal varices or large oesophageal varices (NPV $99.4 \%$ at cut-off value of $3.30 \mathrm{~m} / \mathrm{s}$ ). In comparison, when Bota et al carried out SSM using ARFI technology, they obtained a very good predictive value for cirrhosis (AUROC 0.91), but were not able to distinguish patients with or without oesophageal varices, as well as differences between the grades of oesophageal varices [36].The same author created a formula for the prediction of significant oesophageal varices (at least grade 2) using an original formula with LSM, SSM and the presence of ascites, with reported accuracy of $69.6 \%$ and AUROC 0.721[37].

\section{D-SWE in portal hypertension}

The most recent among elastographic methods that has been evaluated in clinical hepatology is 2D-SWE. 
For this reason very few studies have evaluated this method for the non-invasive assessment of portal hypertension. In two studies, one coming from Spain $(88$ patients) [38] and the other from South Korea (92 patients) [39], the LSM cut-off values for CSPH were15.4 and $15.2 \mathrm{kPa}$, with respective sensitivities of 91.3 and $85.7 \%$, specificities of 90.9 and $80 \%$, and AUROCs of 0.94 and 0.82 .A French group of authors have presented significantly different results for the diagnosis of CSPH with reported LSM cut-off value of $24.5 \mathrm{kPa}$ (sensitivity: $81 \%$, specificity: $83 \%$, AUROC 0.87) [40]. These latest results, although closer to the values accepted for TE, have not proved to be reliable because of the bias spectrum phenomenon: the study included only patients with end-stage cirrhosis on he transplant list, for whom a high LSM was expected, and the majority had CSPH, which is why the statistical power was low [40]. In the above mentioned studies the range of values for the detection of large oesophageal varices was $12.8-16.1 \mathrm{kPa}$ with an AUROC of 0.70-0.89 and with the differing prevalence of large oesophageal varices. As expected, in the French study quoted above significantly higher LSM cut-off values for detecting large oesophageal varices $(24.7 \mathrm{kPa})$ were obtained as well with an AUROC of only 0.6. In light of these results, we are unable to draw reliable conclusions regarding the value of the 2D-SWE in the context of portal hypertension, and further research involving a larger number of patients is required.

An interesting approach to CSPH diagnosis using 2D-SWE was proposed by a group of authors from Bonn, who constructed a sequential algorithm for carrying out LSM and SSM in 158 patients with cirrhosis and a previously established HVPG value [41]. In the first step LSM was performed, and in case of the values $>38 \mathrm{kPa} 36 / 39$ patients were correctly classified with CSPH. In the case of LSM $<38 \mathrm{kPa}$, the next step was to measure SSM values. If the $\mathrm{LSM}$ was $<38 \mathrm{kPa}$ and $\mathrm{SSM}<27.9 \mathrm{kPa}, 32 /$ 40 patients were correctly classified as a group without $\mathrm{CSPH}$, and in case of LSM $<38 \mathrm{kPa}$ but $\mathrm{SSM}>27.9 \mathrm{kPa}$ $35 / 37$ patients were correctly classified with CSPH. This algorithm had a sensitivity of $89.2 \%$ and a specificity of 91.4\% for determining CSPH. However, it should be noticed that spleen stiffness measurement using 2D-SWE, as with other sonoelastographic methods, can be performed with some difficulty in a proportion of patients, with the rate of unreliable results obtained in up to $36.7 \%$ of patients $[42,43]$.

In conclusion, LSM by ultrasound-based elastography can reliably be used to diagnose clinically significant portal hypertension. This refers to the application of the TE method with LSM cut-off values $>25 \mathrm{kPa}$. TE can also reliably exclude the existence of large oesophageal varices in cases of LSM $<20 \mathrm{kPa}$, along with the platelet count $>150 \times 10^{9} / \mathrm{L}$. These recommendations refer primarily to patients with a viral aetiology of chronic liver disease (hepatitis C), while additional studies are needed for other aetiologies. However, based on experience involving the use of TE in fibrosis staging, we can expect LSM cut-off values similar to those mentioned above for the detection of CSPH in other aetiologies of liver disease. Although SSM also poses a logical choice in this indication, controversial results have nevertheless been published on this issue. It should be emphasized, however, that more recent data indicate that this parameter might be included in the diagnostic algorithm for portal hypertension, if not as the sole, then as a part of a sequential algorithm combined with LSM. At this point, given that it has been extensively studied and validated in the context of liver stiffness and portal hypertension, TE could provide a good assessment tool for the hepatological community. Concerning the values of others ultrasound based elastographic methods for $\mathrm{PH}$ evaluation, more scientific papers are necessary to provide their real value, despite the fact that encouraging results have been published.

\section{Conflict of interest: none}

\section{References}

1. Burroughs AK. The Hepatic Artery, Portal Venous System and Portal Hypertension: the Hepatic Veins and Liver in Circulatory Failure. In: Sherlock's Diseases of the Liver and Biliary System. 12th ed. Blackwell Publishing 2011:152-209.

2. Tsochatzis EA, Bosch J, Burroughs AK. Liver cirrhosis. Lancet 2014;383:1749-1761.

3. D'Amico G, Garcia-Tsao G, Pagliaro L. Natural history and prognostic indicators of survival in cirrhosis: a systematic review of 118 studies. J Hepatol 2006;44:217-231.

4. Wongcharatrawee S, Groszmann R. Hemodynamic assessment in clinical practice in portal hypertensive cirrhotics. Ann Gastroenterol 2001;14:158-165.

5. Bosch J, Abraldes JG, Berzigotti A, García-Pagan JC. The clinical use of HVPG measurements in chronic liver disease. Nat Rev Gastroenterol Hepatol 2009;6:573-582.

6. Ripoll C, Groszmann R, Garcia-Tsao G, et al. Hepatic venous pressure gradient predicts clinical decompensation in patients with compensated cirrhosis. Gastroenterology 2007; 133:481-488.

7. Choi SB, Kim HJ, Song TJ, Ahn HS, Choi SY. Influence of clinically significant portal hypertension on surgical outcomes and survival following hepatectomy for hepatocellular carcinoma: a systematic review and meta-analysis. J Hepatobiliary Pancreat Sci 2014;21:639-647. 
8. Berzigotti A, Reig M, Abraldes JB, Bosch J, Bruix J. Portal hypertensionand the outcome of surgery for hepatocellular carcinoma in compensated cirrhosis: a systematic review and meta-analysis. Hepatology 2015;61:526-536.

9. Binţinţan A, Chira RI, Mircea PA. Non-invasive ultrasoundbased diagnosis and staging of esophageal varices in liver cirrhosis. A systematic review of the literature published in the third millenium. Med Ultrason 2013;15:116-124.

10. Bamber J, Cosgrove D, Dietrich CF, et al. EFSUMB guidelines and recommendations on the clinical use of ultrasound elastography. Part 1: Basic principles and technology. U1traschall Med 2013;34:169-184.

11. Cosgrove D, Piscaglia F, Bamber J, et al. EFSUMB guidelines and recommendations on the clinical use of ultrasound elastography. Part 2: Clinical applications. Ultraschall Med 2013;34:238-253.

12. Germani G, Burroughs AK, Dhillon AP. The relationship between liver disease stage and liver fibrosis: a tangled web. Histopathology 2010;57:773-784.

13. European Association for Study of Liver; Asociacion Latinoamericana para el Estudio del Higado. EASL-ALEH Clinical Practice Guidelines: Non-invasive tests for evaluation of liver disease severity and prognosis. J Hepatol 2015;63:237-264.

14. Rosselli M, MacNaughtan J, Jalan R, Pinzani M. Beyond scoring: a modern interpretation of disease progression in chronic liver disease. Gut 2013;62:1234-1241.

15. Garcia-Tsao G, Friedman S, Iredale J, Pinzani M. Now there are many (stages) where before there was one: In search of a pathophysiological classification of cirrhosis. Hepatology 2010;51:1445-1449.

16. Vizzutti F, Arena U, Romanelli RG, et al. Liver stiffness measurement predicts severe portal hypertension in patients with HCV-related cirrhosis. Hepatology 2007;45:12901297.

17. Sandrin L, Fourquet B, Hasquenoph JM, et al. Transient elastography: a new noninvasive method for assessment of hepatic fibrosis. Ultrasound Med Biol 2003;29:1705-1713.

18. Tapper EB, Castera L, Afdhal NH. FibroScan (vibrationcontrolled transient elastography): where does it stand in the United States practice. Clin Gastroenterol Hepatol 2015; 13:27-36.

19. Tsochatzis EA, Gurusamy KS, Ntaoula S, Cholongitas E, Davidson BR, Burroughs AK. Elastography for the diagnosis of severity of fibrosis in chronic liver disease: a metaanalysis of diagnostic accuracy. J Hepatol 2011;54:650659.

20. Shi KQ, Fan YC, Pan ZZ, et al. Transient elastography: a meta-analysis of diagnostic accuracy in evaluation of portal hypertension in chronic liver disease. Liver Int 2013;33:6271.

21. Llop E, Berzigotti A, Reig M, et al. Assessment of portal hypertension by transient elastography in patients with compensated cirrhosis and potentially resectable liver tumors. J Hepatol 2012;56:103-108.

22. Augustin S, Millán L, González A, et al. Detection of early portal hypertension with routine data and liver stiffness in patients with asymptomatic liver disease: a prospective study. J Hepatol 2014;60:561-569.

23. Ding NS, Nguyen T, Iser DM, et al. Liver stiffness plus platelet count can be used to exclude high-risk oesophageal varices. Liver Int 2016;36:240-245.

24. Abraldes JG, Bureau C, Stefanescu H, et al. Noninvasive tools and risk of clinically significant portal hypertension and varices in compensated cirrhosis: The Anticipate study. Hepatology 2016;64:2173-2184.

25. de Franchis R. Expanding consensus in portal hypertension Report of the Baveno VI Consensus Workshop: Stratifying risk and individualizing care for portal hypertension. J Hepatol 2015;63:743-752.

26. Maurice JB, Brodkin E, Arnold F, et al. Validation of the Baveno VI criteria to identify low risk cirrhotic patients not requiring endoscopic surveillance for varices. J Hepatol 2016;65:899-905.

27. Marot A, Trépo E, Doerig C, Schoepfer A, Moreno C, Deltenre P. Liver stiffness and platelet count for identifying patients with compensated liver disease at low risk of variceal bleeding. Liver Int 2016. doi:10.1111/liv.13318.

28. Bolognesi M, Merkel C, Sacerdoti D, Nava V, Gatta A. Role of spleen enlargement in cirrhosis with portal hypertension. Dig Liver Dis 2002;34:144-150.

29. Singh S, Eaton JE, Murad MH, Tanaka H, Iijima H, Talwalkar JA. Accuracy of spleen stiffness measurement in detection of esophageal varices in patients with chronic liver disease: systematic review and meta-analysis. Clin Gastroenterol Hepatol 2014;12:935-945.

30. Stefanescu H, Grigorescu M, Lupsor M, Procopet B, Maniu A, Badea R. Spleen stiffness measurement using Fibroscan for the noninvasive assessment of esophageal varices in liver cirrhosis patients. J Gastroenterol Hepatol 2011;26:164170 .

31. Ma X, Wang L, Wu H, et al. Spleen Stiffness Is Superior to Liver Stiffness for Predicting Esophageal Varices in Chronic Liver Disease: A Meta-Analysis. PLoS One 2016;11:e0165786.

32. Salzl P, Reiberger T, Ferlitsch M, et al. Evaluation of portal hypertension and varices by acoustic radiation force impulse imaging of the liver compared to transient elastography and AST to platelet ratio index. Ultraschall Med 2014;35:528-533.

33. Park Y, Kim SU, Park SY, et al. A novel model to predict esophageal varices in patients with compensated cirrhosis using acoustic radiation force impulse elastography. PLoS One 2015; 10:e0121009.

34. Attia D, Schoenemeier B, Rodt T, et al. Evaluation of Liver and spleen stiffness with acoustic radiation force impulse quantification elastography for diagnosing clinically significant portal hypertension. Ultraschall Med 2015;36:603-610.

35. Takuma Y, Nouso K, Morimoto Y, et al. Measurement of spleen stiffness by acoustic radiation force impulse imaging identifies cirrhotic patients with esophageal varices. Gastroenterology 2013;144:92-101.

36. Bota S, Sporea I, Sirli R, et al. Spleen assessment by Acoustic Radiation Force Impulse Elastography (ARFI) for pre- 
diction of liver cirrhosis and portal hypertension. Med Ultrason 2010;12:213-217.

37. Bota S, Sporea I, Sirli R, et al. Can ARFI elastography predict the presence of significant esophageal varices in newly diagnosed cirrhotic patients? Ann Hepatol 2012;11:519-525.

38. Procopet B, Berzigotti A, Abraldes JG, et al. Real-time shear-wave elastography: applicability, reliability and accuracy for clinically significant portal hypertension. J Hepatol 2015;62:1068-1075.

39. Kim TY, Kim TY, Kim Y, Lim S, Jeong WK, Sohn JH. Diagnostic performance of shear wave elastography for predicting esophageal varices in patients with compensated liver cirrhosis. J Ultrasound Med 2016;35:1373-1381.

40. Elkrief L, Rautou PE, Ronot M, et al. Prospective comparison of spleen and liver stiffness by using shear-wave and transient elastography for detection of portal hypertension in cirrhosis. Radiology 2015;275:589-598.

41. Jansen C, Bogs C, Verlinden W, et al. Shear-wave elastography of the liver and spleen identifies clinically significant portal hypertension: A prospective multicentre study. Liver Int 2016;37:396-405.

42. Grgurevic I, Puljiz Z, Brnic D, et al. Liver and spleen stiffness and their ratio assessed by real-time two dimensional-shear wave elastography in patients with liver fibrosis and cirrhosis due to chronic viral hepatitis. Eur Radiol 2015;25:3214-3221.

43. Cassinotto C, Charrie A, Mouries A, et al. Liver and spleen elastography using supersonic shear imaging for the noninvasive diagnosis of cirrhosis severity and oesophageal varices. Dig Liver Dis 2015;47:695-701. 